\title{
Patrones de titubeo en el habla espontánea de estudiantes de ELE húngaros: la influencia de la inmersión en el país meta
}

\author{
Kata Baditzné Pálvölgyi \\ Universidad Eötvös Loránd, Budapest \\ b.palvolgyi.kata@btk.elte.hu
}

\section{Resumen}

Según investigaciones previas (BADITZNÉ, 2019c), al escuchar el habla espontánea de estudiantes de ELE húngaros de nivel umbral (B1 según el MCER), uno de los aspectos que menos toleraban los hablantes nativos de español era la forma en que los húngaros titubeaban en español, por lo tanto, en este estudio nos enfocamos principalmente en fenómenos de disfluencia como las pausas sonoras y los alargamientos, recursos frecuentes de la hesitación. En la investigación primero nos centraremos en la forma de titubear de 20 estudiantes de ELE húngaros: un grupo de 10 alumnos que todavía no han vivido en España, y otro de una decena de alumnos en el que todos han pasado algún tiempo en el país meta, y luego contrastamos sus patrones de titubeo con las pautas empleadas por 25 españoles nativos. Como era de esperar, la estancia en España influyó positivamente en ciertos aspectos de la adquisición de los esquemas de titubeo nativos, por ejemplo el tiempo gastado en fenómenos de disfluencia disminuyó notablemente, pero el timbre vocálico de las pausas llenas no dejó de ser un ámbito de transferencia negativa de la lengua materna de los alumnos.

Palabras clave: Hesitación. Pausas sonoras. Alargamientos. Transferencia negativa. Nivel umbral.

\footnotetext{
Abstract

According to previous research (BADITZNÉ, 2019c), when listening to the spontaneous speech of Hungarian students of Spanish at threshold level (B1 of CEFR), one of the aspects that native Spanish speakers least tolerated was the way in which Hungarians hesitated in Spanish, therefore in this study we are focusing mainly on disfluency phenomena such as filled pauses and lengthenings, which are frequent hesitation devices. In the investigation we will first focus on the hesitation patterns employed by 20 Hungarian students of Spanish: a group of 10 students who have not yet lived in Spain, and another of 10 students who all have spent some time
} 
in the target country, and then we contrast their hesitation patterns with those employed by 25 native Spanish speakers. As expected, the stay in Spain positively influenced certain aspects of the acquisition of native hesitation schemes, for example, the overall duration of disfluency phenomena decreased considerably, but the vocalic timbre of filled pauses still seems to be a challenging area of negative transfer.

Keywords: Hesitation. Filled pauses. Lengthenings. Negative transfer. Threshold level.

\section{Introducción}

El tema de la disfluencia de la lengua materna se ha discutido ampliamente en numerosos estudios, pero los fenómenos de la disfluencia L2 deberían merecer más atención en proyectos de investigación (RIEGER, 2003) y también en el aula (BELZ et al., 2017). Según Medgyes (2001), hay ciertas áreas que, aunque los aprendices de idiomas permanezcan en el país meta del idioma aprendido durante mucho tiempo, no pueden dominarlas a la perfección. Estas áreas incluyen el vocabulario, las expresiones idiomáticas, la destreza de la comprensión auditiva, la fluidez y la pronunciación. Si nos concentramos en estos dos últimos, vemos que el titubeo está conectado a ambos ámbitos. Los sonidos que empleamos cuando titubeamos no son necesariamente universales; y, si definimos el titubeo como la interrupción del habla continua, lo vemos como un bloqueador de la fluidez. Por otra parte, el titubeo también puede garantizar que el hablante mantenga el turno del diálogo, o al menos atraer la atención de los oyentes (RUTGER BOSKER et al., 2015), por lo que tiene un papel doble en el discurso oral. La cuestión de los turnos es de gran importancia en el español, ya que esta lengua se caracteriza por reglas aparentemente violentas relacionadas con las técnicas de debate, que incluso pueden causar un choque cultural (BERRY, 1994), por ejemplo, a los húngaros, que no están acostumbrados al llamado 'debate mediterráneo'. Las formas de mantener y, especialmente, obtener turnos conversacionales parecen demasiado vehementes para un extranjero, y si los estudiantes de español no están equipados con las estrategias adecuadas, pueden perder terreno en las conversaciones espontáneas con hablantes nativos de español, lo que obviamente no es su objetivo. 
Por lo tanto, debemos prestar especial atención a proveer a los estudiantes de español de los recursos necesarios para que sean participantes seguros de sí mismos en las conversaciones y debates en español. Como hemos mencionado, los esquemas de titubeo pueden ser la clave para asegurar que el hablante pueda mantener un turno conversacional. Por eso es realmente importante que los estudiantes de idiomas adquieran los patrones de titubeo correctos cuando aprenden el idioma meta. Pero, ¿aprenden los húngaros a titubear en español? Incluso a nivel avanzado, aparentemente no; una investigación previa (BADITZNÉ, 2019c) mostró que los hablantes nativos de español toleraban menos los siguientes aspectos en el habla espontánea en español de los estudiantes de lengua materna húngara (de nivel B1 según el Marco Común de Referencia para las Lenguas como mínimo): la pronunciación de ciertas vocales y consonantes (en su mayoría sibilantes); la inadecuación de los patrones de entonación empleados; la lentitud del ritmo del habla, así como la forma en que los estudiantes húngaros titubeaban.

Por lo tanto, conviene examinar concretamente cuáles son los factores que llevaban a los hispanohablantes criticar este último aspecto, "la forma en que los húngaros titubean" en la expresión oral de los aprendientes húngaros de ELE.

La hesitación se considera un fenómeno de disfluencia. Existen varias subcategorizaciones relacionadas con los fenómenos de disfluencia (NEUBERGER, 2014, p. 23), pero nosotros elegimos la de Gósy (2002). Ella categoriza los fenómenos de disfluencia en dos grupos: las realizaciones erróneas y los fenómenos relacionados con la inseguridad del hablante. Este segundo grupo incluye las pausas silenciosas, las pausas llenas, repeticiones, alargamientos y falsas partidas. Debido a que tanto las pausas llenas como los alargamientos pueden considerarse 'titubeos' en una terminología no académica, en nuestra investigación nos centramos en estos dos fenómenos de disfluencia.

En Baditzné (2019a) vimos que respecto a la frecuencia y la duración de los fenómenos de disfluencia, los húngaros titubean mucho más que los españoles ${ }^{1}$. Hemos comparado la producción oral de 25 españoles de Oviedo

${ }^{1}$ Los húngaros titubeaban durante el $16.93 \%$ de su tiempo total de habla en comparación con el $10.37 \%$ en el caso de los informantes españoles. Estos son 
y de 25 estudiantes húngaros de ELE en la misma tarea, y resultó que la duración del tiempo aplicado en fenómenos de disfluencia respecto al tiempo total del habla era superior en el caso de los húngaros (casi el doble), y esta diferencia se debe más bien a la frecuencia de los fenómenos de titubeo que a su duración, ya que el tiempo medio de los titubeos y alargamientos es prácticamente idéntico en el caso de los aprendientes húngaros de ELE y los españoles nativos, mientras que los aprendientes húngaros titubeaban más veces al minuto que los españoles. Por lo tanto, ya sabemos que la relativamente alta frecuencia de los fenómenos de disfluencia es un aspecto que puede contribuir al juicio negativo de los españoles sobre la manera de titubear de los estudiantes de ELE hungaroparlantes.

Además de contrastar los datos relacionados con la frecuencia y la duración de los segmentos afectados por el titubeo, en Baditzné (2019b) hemos ampliado nuestro enfoque con un aspecto más: las características fónicas del titubeo en el habla espontánea de los estudiantes de ELE húngaros. Este ámbito es importante ya que si examinamos las diferencias entre el húngaro y el español en lo que respecta a las técnicas de titubeo, encontramos que hay una gran diferencia: mientras que los húngaros usan principalmente el schwa y el sonido [m] para titubear (Horváth, 2014), el español aplica el sonido español [e] (MACHUCA et al., 2015), así como el sonido [a] y el sonido [m] (GARRIDO et al., 2017). Esto implica que el hecho de que los húngaros titubeen de manera inusual según la percepción

valores medios en el caso de los 25-25 informantes, y las pruebas estadísticas (prueba t de 1 cola para la diferencia de medias) revelaron que existía una diferencia estadística entre las medias en el intervalo de confianza del 95\% ( $<<0,05)$. En cuanto a la frecuencia de los titubeos, los valores medios en ambos corpus fueron diferentes, por lo que aplicamos nuevamente una prueba t de una cola que demostró que la diferencia de las dos medias (20,21 fenómenos por minuto en el caso de los húngaros comparado con 11,22 en el corpus español) era estadísticamente diferente en el intervalo de confianza del 95\% ( $\mathrm{p}<0,05)$. La duración media de los fenómenos de titubeo, por otro lado, no pareció ser diferente en los dos grupos: sorprendentemente, los húngaros no produjeron alargamientos más largos en comparación con los hablantes nativos de español. La duración media de una hesitación o alargamiento en el caso de los hispanohablantes fue de 0,55 segundos y 0,51segundos en el caso de los hablantes de húngaros, cuya diferencia, según la prueba t de dos colas para la igualdad de medias resultó no ser estadísticamente significativa en el intervalo de confianza del 95\% ( $\mathrm{p}=0,058 ; \mathrm{p}>0,05)$. 
de los españoles nativos, puede estar vinculado, además de la frecuencia demasiado alta de los fenómenos de titubeo, a la forma en que titubean en español: tienden a emplear los sonidos usados en húngaro para titubear a consecuencia de una transferencia negativa de la lengua materna. Esta suposición se verificó en nuestras investigaciones: según Baditzné (2019b), los aprendientes de ELE húngaros efectivamente empleaban en las pausas llenas las vocales que según su estructura formántica se situaban más bien dentro de los márgenes de dispersión de los sonidos [ə] y [œ], y no de los sonidos [e] o [a] que serían los empleados por españoles nativos.

Un aspecto que vale la pena considerar - y que vamos a exponer en el presente estudio -es una comparación entre estudiantes húngaros que aprenden español en aulas de idiomas extranjeros en un contexto de no inmersión y estudiantes que aprenden español en el extranjero. La estancia en el país meta - en nuestro caso, España - iinfluye de manera considerable en los esquemas de titubeo de los estudiantes de ELE húngaros? De acuerdo con los resultados de García-Amaya (2015) en el caso de aprender español como idioma extranjero, el contexto del estudio de inmersión produjo una mayor fluidez y mejora de la competencia lingüística, pero también un aumento en el uso de pausas llenas, por ejemplo. Nosotros, sin embargo, esperamos que por la estancia en el país meta los alumnos de ELE reduzcan los fenómenos de disfluencia, y se acerquen a los patrones españoles nativos de titubeo tanto en la cantidad como en la cualidad.

Como hipótesis de investigación, formularemos las siguientes presuposiciones:

Los estudiantes de ELE húngaros que pasaron algún tiempo en España, se acercarán más a los patrones de titubeo de los españoles nativos en los siguientes aspectos:

1) la duración del tiempo gastado en fenómenos de disfluencia;

2) la duración media de los fenómenos de disfluencia;

3) la cantidad de los fenómenos de disfluencia;

4) el timbre vocálico de las pausas llenas.

Para confirmar nuestras hipótesis, llevamos a cabo una investigación de dos fases. Primero, observaremos los patrones de titubeo en dos corpus de estudiantes de ELE húngaros: uno con informantes que no habían estado todavía en el país meta y otro con alumnos que sí pasaron una temporada en España. Después, en la segunda fase, los resultados obtenidos se contrastarán 
con los datos que poseemos tras analizar las formas de titubeo en un corpus de informantes de Oviedo (BADITZNÉ, 2019a).

\section{El corpus}

Para validar nuestras hipótesis, recopilamos datos de estudiantes húngaros de ELE y contrastamos los resultados con los datos obtenidos de un corpus de hablantes nativos de español. Nuestro corpus de españoles nativos que sirvió de referencia consistió en grabaciones de audio provenientes de 25 hispanohablantes del norte de España (de Asturias, de 18 a 23 años, todos aprendiendo Humanidades en la Universidad de Oviedo; 7 hombres y 18 mujeres). El corpus de estudiantes de ELE húngaros proviene de 20 estudiantes de español húngaros (de Budapest, de 19 a 25 años, todos aprendiendo Filología Española en la Universidad Eötvös Loránd; 1 hombre y 19 mujeres). En cuanto al nivel de dominio de español de los estudiantes húngaros, todos alcanzaron al menos el nivel B1 ("umbral") de acuerdo con el MCERL, pero los 10 estudiantes del primer grupo no han vivido todavía en ningún país hispanohablante; en el caso de los 10 informantes del segundo grupo, los alumnos pasaron como mínimo un mes en España y como máximo, un año (los estudiantes que habían vivido en América Latina fueron excluidos del corpus).

Las grabaciones fueron realizadas por Zoltán Kristóf Gaál (Universidad ELTE), que entrevistó a los informantes en el marco de una actividad de Map Task (los entrevistados tenían que informar al entrevistador sobre el itinerario correcto de acuerdo con un mapa). De esta manera pudimos garantizar que el discurso grabado fuera espontáneo o por lo menos semiespontáneo y, por lo tanto, adecuado para un análisis desde la perspectiva de los fenómenos de disfluencia.

\section{Método}

En nuestro corpus examinamos los siguientes aspectos:

1) la duración del tiempo empleado en fenómenos de disfluencia (en caso de alargamientos y pausas llenas, comparando con el tiempo del habla total);

2) la duración media de los fenómenos de disfluencia, en segundos; 
3) la cantidad de los fenómenos de disfluencia (por minuto);

4) el timbre vocálico de las pausas llenas (los dos primeros formantes de las vocales).

La anotación se realizó en base a la información extraída del software de análisis acústico Praat, teniendo en cuenta el siguiente principio: al medir el alargamiento, siempre tomamos la sílaba como el dominio básico del análisis. Esto fue así en el caso del fenómeno típico español de resilabificación (es decir, cuando una consonante final de palabra se resilabifica como el inicio de la siguiente palabra que comienza con una vocal, como en [la: s alondras], la longitud de la sílaba solo se midió para [la:], ya que la s fue resilabificada a la sílaba siguiente a- en alondras.

\section{Resultados}

Primero nos enfocaremos en la duración y la frecuencia de los fenómenos de disfluencia en ambos grupos, comparando los resultados con los del grupo de referencia (los informantes españoles nativos). En cuanto a la duración, primero se proporcionan los datos medios en segundos de todos los fenómenos de disfluencia hallados en los tres corpus. Después, veremos el tiempo empleado en fenómenos de disfluencia comparando con el tiempo total de habla (valores medios, en segundos), y finalmente se expondrá el número medio de los fenómenos de disfluencia encontrados por minuto en los tres corpus. La tabla 1. resume los resultados.

Como se desprende de la tabla, los estudiantes que pasaron algún tiempo en España se acercan más a la realización española en dos aspectos: titubean con menos frecuencia que sus compañeros sin experiencia en el país meta y en su habla invierten menos tiempo en los fenómenos de disfluencia. Podemos constatar de los valores medios que tanto el tiempo dedicado a los fenómenos de disfluencia como la duración de los fenómenos de disfluencia no son significativamente diferentes en el caso de los nativos y los húngaros que han vivido ya en España. Este último aspecto - la duración media de los fenómenos de disfluencia - es prácticamente idéntica en los tres grupos. De esta forma, en cuanto a la cantidad de los fenómenos de disfluencia, podemos sacar las siguientes conclusiones:

Los estudiantes de ELE húngaros, tras vivir alguna temporada en España, efectivamente titubean menos que los estudiantes de ELE húngaros 
sin esta experiencia, y de esta forma se acercan a las características del titubeo de los nativos. La duración de los fenómenos de disfluencia, en cambio, no parece ser un factor relevante, ya que no hay una diferencia significativa ni entre los dos grupos húngaros, ni entre estos y los españoles nativos.

Tabla 1. Las características de la cantidad de los fenómenos de disfluencia.

\begin{tabular}{|c|c|c|c|}
\hline & $\begin{array}{c}\text { estudiantes de ELE } \\
\text { húngaros sin } \\
\text { estancia en España }\end{array}$ & $\begin{array}{c}\text { españoles } \\
\text { nativos }\end{array}$ & $\begin{array}{c}\text { estudiantes } \\
\text { de ELE } \\
\text { húngaros } \\
\end{array}$ \\
\hline $\begin{array}{l}\text { duración media de los } \\
\text { fenómenos de disfluencia } \\
\text { (sec) }\end{array}$ & 0,52 & 0,55 & 0,53 \\
\hline $\begin{array}{l}\text { resultados de la prueba t de } \\
\text { dos colas asumiendo } \\
\text { igualdad de medias (ig. de } \\
\text { varianzas) }\end{array}$ & $\begin{array}{r}\mathrm{p}>0,05 \\
\text { no diferencia } \\
\text { significativa }\end{array}$ & & $\begin{array}{r}\mathrm{p}>0,05 \\
\text { no } \\
\text { diferencia } \\
\text { significativa }\end{array}$ \\
\hline $\begin{array}{l}\text { tiempo invertido en } \\
\text { fenómenos de disfluencia } \\
\text { comparando con el tiempo } \\
\text { de habla total (sec) }\end{array}$ & 20,2 & 10,37 & 13,77 \\
\hline $\begin{array}{l}\text { resultados de la prueba t de } \\
\text { una cola asumiendo } \\
\text { diferencia de medias (ig. } \\
\text { de varianzas) }\end{array}$ & $\begin{array}{r}\mathrm{p}<0,05 \\
\text { diferencia } \\
\text { significativa }\end{array}$ & & $\begin{array}{r}\mathrm{p}>0,05 \\
\text { no } \\
\text { diferencia } \\
\text { significativa }\end{array}$ \\
\hline $\begin{array}{l}\text { número medio de } \\
\text { fenómenos de disfluencia } \\
\text { por minuto }\end{array}$ & 24,32 & 11,22 & 15,96 \\
\hline $\begin{array}{l}\text { resultados de la prueba t de } \\
\text { una cola asumiendo } \\
\text { diferencia de medias (ig. } \\
\text { de varianzas) }\end{array}$ & $\begin{array}{r}\mathrm{p}<0,05 \\
\text { diferencia } \\
\text { significativa }\end{array}$ & & $\begin{array}{r}\mathrm{p}<0,05 \\
\text { diferencia } \\
\text { significativa }\end{array}$ \\
\hline
\end{tabular}

Fuente: Autoría propia

A continuación, si examinamos la cualidad de los fenómenos de disfluencia, podemos notar unas diferencias relevantes que se resumen en las 
siguientes figuras. Para exraer los valores de los primeros dos formantes característicos de las pausas llenas, se ha utilizado el software de análisis acústico Praat (BOERSMA; WEENINK, 2019; mediante las funciones "get first formant" y "get second formant", respectivamente, que exponen los valores medios característicos del segmento seleccionado). En cuanto a la distribución de los primeros dos formantes, podemos ver en la Figura 1 que los húngaros - independientemente de que si han pasado algún tiempo en España o no - tienden a realizar diferentes sonidos vocálicos que los informantes españoles:

Figura 1. La realización de las pausas llenas: formantes vocálicos de los tres grupos

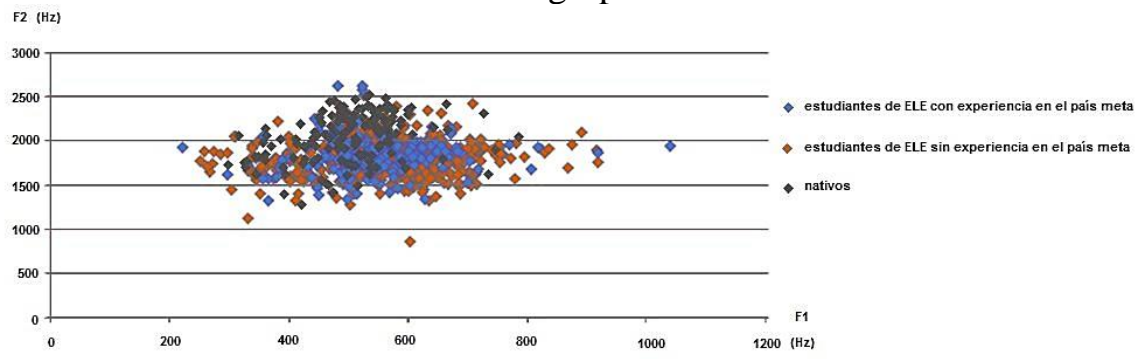

Fuente: Autoría propia

La Figura 2, además de la información que proporciona la Figura 1, muestra la posición de las vocales cardinales (DELATTRE et al., 1952) completada por las vocales húngaras [ع], [e:], [œ], [ø:], la schwa en color verde (GÓSY, 2004 citado por AUSZMANN, 2018: 30) y las vocales españolas [e] y [a] en rojo (QUILIS, 1999: 163), en comparación con lo que hemos encontrado en nuestros corpus. Según se visualiza en la Figura 2, en las pausas llenas, el sonido más típico en el corpus español es [e] o $[\varepsilon]$, mientras que en la interlengua húngaro-española, [ə] o [œ]. 
Figura 2. La realización de las pausas llenas: formantes vocálicos de los tres

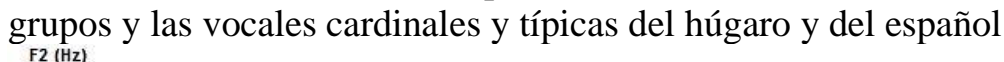

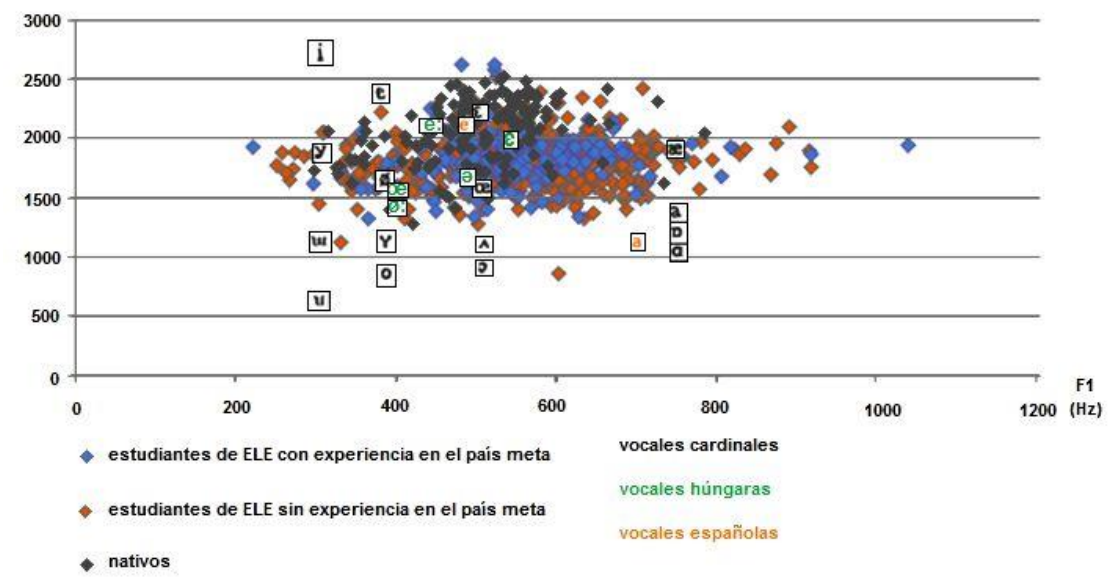

Fuente: Autoría propia

Como conclusiones respecto a la cualidad de los fenómenos de disfluencia, es observable que mientras que los dos primeros formantes de los hablantes españoles se realizaron cerca o dentro de los márgenes de dispersión de los sonidos [e] o [ع], los estudiantes húngaros típicamente produjeron los sonidos [ə] o [œ]. Es interesante observar que también fue característico pronunciar un schwa alargado al final de las palabras en lugar de alargar una vocal o una consonante en la palabra afectada, como en [e: 1] (la realización nativa en español del artículo determinado masculino) en contraste con la solución de los estudiantes húngaros [elə:].

\section{Conclusión y discusión}

Como una reflexión sobre nuestra hipótesis, hemos visto que, en comparación con los patrones de hesitación nativos de español, los estudiantes húngaros de español que vivieron en España:

1) no producen segmentos alargados considerablemente más largos que los españoles, 
2) no titubean con una frecuencia considerablemente más alta,

3) pero en las realizaciones vocálicas de los titubeos, se acercan más a sus patrones nativos.

En cuanto a los estudiantes de ELE húngaros que no vivieron en España, podemos observar que:

1) no producen segmentos alargados considerablemente más largos que los españoles,

2) pero titubean considerablemente más a menudo,

3) y en las realizaciones vocálicas de los titubeos, se acercan más a sus patrones nativos.

Eso significaría que una estancia en el extranjero sí puede reducir la cantidad de tiempo empleado en la hesitación, pero parece ser insuficiente en sí para que los alumnos produzcan los patrones vocálicos característicos del español en cuanto a los fenómenos de disfluencia. Nuestros resultados plantean algunas preguntas acerca de la adquisición de los esquemas de titubeo españoles: ¿Cómo lograr que los estudiantes de ELE se acerquen más a las realizaciones vocálicas características del titubeo de la lengua meta? ¿Sería necesaria una estancia más alargada, superior a un año? ¿O es la calidad de la estancia (por ejemplo, el mayor grado de inmersión en el contexto español) que posibilita la reproducción de los patrones españoles? ¿Pueden ser una solución las prácticas concentradas en esta área concreta, tras una concienciación sobre la transferencia negativa del patrón húngaro? ¿Cómo fomentar la adquisición de los patrones de hesitación naturales de la lengua meta cuando la inmersión no es una solución viable?

Naturalmente, nuestra investigación se basa en la interpretación de datos provenientes únicamente del español del norte, por lo que en el futuro se recomiendan estudios adicionales para analizar también los fenómenos de hesitación en los dialectos del sur del español.

Con respecto a los patrones de duración de los titubeos independiente del hablante, en el futuro, es conveniente atenerse a un modelo objetivo para ver cómo se realiza el alargamiento en comparación con su contexto. La estandarización de la duración nos permitiría ver hasta qué punto una sílaba alargada es más larga que otros intervalos silábicos dentro de la misma expresión; para una propuesta al respecto, véase por ejemplo el modelo de análisis prosódico de Cantero (2019). El manejo de la duración relativa podría ayudarnos a generalizar mejor que si simplemente comparáramos los datos de duración absoluta. 


\section{Expresiones de gratitud}

Nos gustaría expresar nuestro sincero agradecimiento a Zoltán Kristóf Gaál, estudiante de doctorado del Programa de Doctorado en Lingüística Románica de la Universidad Eötvös Loránd, en Budapest, por proporcionarnos las grabaciones que utilizamos para nuestro corpus. Al mismo tiempo agredecemos a László Pálvölgyi y a Péter Pálvölgyi sus valiosos comentarios sobre la interpretación de los datos.

El presente estudio se ha realizado gracias al apoyo del "Nuevo Programa Nacional de Excelencia" ÚNKP-19-4 del Departamento Nacional de Investigación, Desarrollo e Innovación de Hungría.

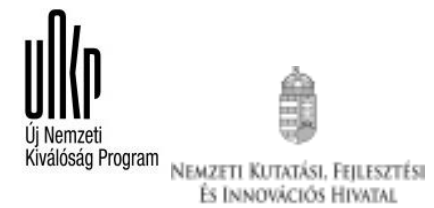

\section{Referências}

AUSZMANN, A. Magyar gyermekek magánhangzóinak akusztikaifonetikai jellemzői. Beszéd. Kutatás. Alkalmazás. Eötvös Loránd Tudományegyetem: Eötvös Kiadó, 2018.

BADITZNÉ PÁLVÖLGYI, K. Hesitation patterns in the Spanish Spontaneous Speech of Hungarian learners of Spanish. In: ROSE, R. L; EKLUND, R. (Eds.) Proceedings of DiSS 2019, 12-14 September 2019, Eötvös Loránd University, Budapest, Hungary, 2019a, p. 35-38.

BADITZNÉ PÁLVÖLGYI, K. A hezitálás mintázatai küszöbszinten álló magyar ajkú nyelvtanulók spontán beszédprodukciójában. Alkalmazott Nyelvtudomány 19:2, 2019b, p. 1-14.

BADITZNÉ PÁLVÖLGYI, K. Magyar ajkú spanyol nyelvtanulók kiejtése spanyol anyanyelvüek szemével. XXVII. Magyar Alkalmazott Nyelvészeti Kongresszus. Nyelv, kultúra, identitás. Alkalmazott nyelvészeti kutatások a 21. századi információs térben. KRE, Budapest, 2019c. április 15-16. Absztraktfüzet. p. 44. 
BELZ, M. et al. Fluently disfluent? Pauses and repairs of advanced learners and native speakers of German. International Journal of Learner Corpus Research 3:2, 2017, p. 118-148.

BERRY, A. Spanish and American turn-taking styles. In: Pragmatic and Language Learning. Monograph Series, Volume 5, 1994, p. 180-190.

BOERSMA, P.;WEENINK, D. Praat. Doing phonetics by computer. Version 6.0.49, 2019.

CANTERO SERENA, F. J. Análisis prosódico del habla: más allá de la melodía, In: ÁLVAREZ SILVA, M. R.; MUÑOZ ALVARADO, A.; RUIZ MIYARES, L. (Eds.): Comunicación Social: Linguiística, Medios Masivos, Arte, Etnología, Folclor y otras ciencias afines. Volumen II. Santiago de Cuba: Ediciones Centro de Linguiística Aplicada, 2019, p. 485498.

GARRIDO ALMIÑNAN, J. M.; LAPLAZA, Y.; GARCÍA, C. L. 2017. La caracterización pragmática y prosódica de la vocalización "mmm" en español. In: MARRERO AGUIAR, V.; ESTEBAS VILAPLANA, E. (Eds.): Tendencias actuales en fonética experimental: Cruce de disciplinas en el centenario del Manual de Pronunciación Española (Tomás Navarro Tomás), Madrid: Uned, 2017, p. 125-129.

GARCÍA-AMAYA, L. A longitudinal study of filled pauses and silent pauses in second language speech. In: EKLUND, R. (Ed.) Papers presented at Diss 2015. Queen Margaret University, Edinburgh, 2015, p. 23-27.

GÓSY, M. A megakadásjelenségek eredete a spontán beszédtervezés folyamatában. Magyar Nyelvőr 126, 2002. p. 192-204.

HORVÁTH, V. Hezitációs jelenségek a Magyar beszédben. Beszéd. Kutatás. Alkalmazás. Eötvös Loránd Tudományegyetem: Eötvös Kiadó, 2014.

MACHUCA, M. J.; LLISTERRI, J.; RÍOS, A. Las pausas sonoras y los alargamientos en español. Unestudiopreliminar. Revista Normas, 5, 2015, p. 81-96.

MEDGYES, P. When the teacher is a non-native speaker. Teaching pronunciation, 2001, p. 429-442.

NEUBERGER, T. A spontán beszéd sajátosságai gyermekkorban. 
Patrones de titubeo en el habla espontánea...

Beszéd. Kutatás. Alkalmazás. Eötvös Loránd Tudományegyetem: Eötvös Kiadó, 2014.

RIEGER, C. L. Disfluencies and hesitation strategies in oral L2 tests. Proceedings of DiSS'03, Disfluency in Spontaneous Speech Workshop, 5-8 September 2003, Göteborg University, Sweden. Robert EKLUND (Ed.), Gothenburg Papers in Theoretical Linguistics n. 90, 2003. p. 4144.

RUTGER BOSKER, H. et al. Both native and non-native disfluencies trigger listeners' attention. In: EKLUND, R. (Ed.) Papers presented at Diss 2015. Queen Margaret University: Edinburgh, 2015. p. 5-8.

Recibido en: 06/12/2019

Aceptado en: 28/05/2020

Title: Hesitation patterns in the spontaneous speech of Hungarian learners of Spanish: the influence of immersion in the target country. 